Article

\title{
Luminescent and UV-Shielding ZnO Quantum Dots/Carboxymethylcellulose Sodium Nanocomposite Polymer Films
}

\author{
Tianyi Li, Bin Li, Yali Ji and Lili Wang * \\ College of Science, Northeast Forestry University, Harbin 150040, China; 18845562646@163.com (T.L.); \\ libinrainkiss1014@163.com (B.L.); jyl1513062809@163.com (Y.J.) \\ * Correspondence: rainkiss1014@nefu.edu.cn; Tel.: +86-451-8219-0405
}

Received: 7 September 2018; Accepted: 3 October 2018; Published: 8 October 2018

\begin{abstract}
Luminescent and UV-shielding bio-polymers have seldom been reported. Zinc oxide quantum dots (ZnOQD) $(\sim 3.2 \mathrm{~nm})$ was synthesized by a short-term sol-gel method. ZnOQD possessed a crystal lattice spacing of $0.28 \mathrm{~nm}$ and a hexagonal wurtzite structure. Luminescent and UV-shielding ZnOQD/carboxymethylcellulose sodium (CMC) nanocomposite polymer films were successfully fabricated by incorporating ZnOQD into a CMC matrix through a solution casting method. Thermal analysis demonstrated that the $\mathrm{ZnOQD}$ reduce the thermal decomposition rate of $\mathrm{CMC}$, and a large number of ZnOQD can promote the catalytic degradation of $\mathrm{ZnOQD/CMC} \mathrm{nanocomposites.}$ Furthermore, ZnOQD/CMC hybrid polymer films exhibited photoluminescence with maximum emission wavelength at $525 \mathrm{~nm}$. More significantly, ZnOQD/CMC showed prominent UV-absorbing capability. Such ZnOQD/CMC nanocomposite polymer films are promising in UV-shielding and optical applications.
\end{abstract}

Keywords: UV-shielding quantum dots; biomaterials; luminescent; polymer; nanocomposite

\section{Introduction}

Recently, many composites have been reported and have aroused current interest [1,2]. In particular, many natural bio-polymers have been widely used in packaging [3,4] and within the medical industry [5,6] due to their biocompatibility [7], biodegradability [8], low toxicity and edibility $[9,10]$. Carboxymethylcellulose sodium (CMC) as a biopolymer has high optical transparency, good chemical resistance and film forming properties. More importantly, CMC is rich sources and sustainable. However, luminescent and UV-shielding CMC-matrix nanocomposites have seldom been reported.

$\mathrm{ZnO}$ quantum dots (ZnOQD) with a wide band gap of $3.37 \mathrm{eV}$ and a rather large exciton binding energy of $60 \mathrm{meV}$ exhibit unique optical and electrical properties [11] due to the quantum confinement effect [12,13]. ZnOQD has the merits of being non-toxic [14], water dispersibility [15] and biocompatibility [16]. Therefore, in the present work, ZnOQD was synthesized using a short-term sol-gel method. The morphology, particle size, crystalline structure, and thermal and optical properties were characterized using an enlarged high-resolution transmission electron microscope (HRTEM), selected area electron diffraction (SAED), powder X-ray diffraction (XRD) pattern, thermogravimetric analysis, ultraviolet-visible (UV-vis) and photoluminescence spectra, respectively. Furthermore, to obtain CMC-based luminescent polymer hybrid films, ZnOQD/CMC nanocomposites were successfully prepared via a green simple procedure. The optical and UV-shielding properties of ZnOQD/CMC composites were investigated. This work broadened the applications of biopolymers in UV-shielding [17] and within the optical field. 


\section{Materials and Methods}

\subsection{Materials}

Zinc acetate (99\%), lithium hydroxide (99\%) and carboxymethylcellulose sodium (CMC) (99\%) were purchased from Tianjin Kermiou Chemical Reagent Co. Ltd. (Tianjin, China). Ethanol (99\%) was purchased from Tianjin Fuyu Fine Chemical Co. Ltd. (Tianjin, China). All the reagents and chemicals were of analytical grade without further purification. Deionized water (Milli-Q Academic, Beijing, China) was used throughout the whole experiment.

\subsection{Preparation of $\mathrm{ZnOQD}$ and $\mathrm{ZnOQD/CMC} \mathrm{Nanocomposites}$}

Ethanol was chosen as solvent. Firstly, $0.878 \mathrm{~g}(4 \mathrm{mmol})$ of zinc acetate and $0.2672 \mathrm{~g}(6 \mathrm{mmol})$ of lithium hydroxide were dissolved in $100 \mathrm{~mL}$ of ethanol, respectively. Secondly, the above two solutions were ultrasonicated at $70^{\circ} \mathrm{C}$ for $20 \mathrm{~min}$. Thirdly, prior to the addition of lithium hydroxide solution, zinc acetate solution was refluxed at $78{ }^{\circ} \mathrm{C}$ for $15 \mathrm{~min}$ under continuous stirring. Then the mixed solution reacted by further refluxing with stirring under $78^{\circ} \mathrm{C}$ for $25 \mathrm{~min}$. The as-prepared zinc oxide quantum dots were dried in the oven at $70{ }^{\circ} \mathrm{C}$ for $2 \mathrm{~h}$ and the resulting sample was labeled as ZnOQD.

\subsection{Fabrication of $\mathrm{ZnOQD/CMC} \mathrm{Composites}$}

Three grams of CMC were dissolved in $100 \mathrm{~mL}$ of distilled water under stirring for $4 \mathrm{~h}$ at $60{ }^{\circ} \mathrm{C}$. $\mathrm{ZnOQD}$ was dispersed in $20 \mathrm{~mL}$ of distilled water treated by ultrasonic for $1 \mathrm{~h}$. Then ZnOQD and $\mathrm{CMC}$ aqueous solution were mixed. The mixtures were casted onto glass substrates with no post treatment. Furthermore, the glass substrates were transferred to the oven and dried for $10 \mathrm{~h}$ at $60{ }^{\circ} \mathrm{C}$ to obtain ZnOQD/CMC films. The ZnOQD/CMC composites with 5, 10 and 15 wt \% loading of ZnOQD were fabricated, respectively. Thus, the resulting ZnOQD/CMC films were labeled as $5 \mathrm{wt} \%$ QD-CMC, 10 wt \% QD-CMC and 15 wt \% QD-CMC, respectively.

\subsection{Characterization Techniques}

The specimens were examined in a TECNAI 10 PHILIPS transmission electron microscope (TEM) (Amsterdam, Holland) operated at the accelerating voltage of $100 \mathrm{kV}$. The powder X-ray diffraction (XRD) pattern of the samples was recorded by a D/MAX 2200 diffractometer (Rigaku, Tokyo, Japan) using $\mathrm{Cu} \mathrm{K} \alpha$ radiation in a scan step of $0.02^{\circ}$ and a scan range between $5^{\circ}$ and $70^{\circ}$. All thermogravimetric analysis (TGA) tests were carried out by a thermal analyzer (TGA pyris 1, Perkin Elmer Co., Waltham, MA, USA) at a linear heating rate of $10^{\circ} \mathrm{C} / \mathrm{min}$ under pure nitrogen within the temperature range of 50 to $800^{\circ} \mathrm{C}$. The mass of the samples was kept within $3-4 \mathrm{mg}$. Ultraviolet-visible (UV-vis) and photoluminescence spectra of ZnOQDs and ZnOQD/CMC nanocomposites were recorded on a GENESYS $10 \mathrm{UV}$-vis spectrometer (Beijing, China) and fluorescence F-4600 fluorescence spectro-photometer (Hitachi, Japan), respectively.

\section{Results and Discussion}

\subsection{Morphology and Diffraction Analysis of $\mathrm{ZnOQD}$}

Figure 1a shows TEM images of ZnOQD featured spherical particles along with good monodispersity. Figure $1 \mathrm{~b}$ displays the size distribution histogram measured from 100 particles. As presented, the average size of ZnOQD is (3.2 \pm 0.2$) \mathrm{nm}$. Figure 1c gives the enlarged high-resolution transmission electron microscope (HRTEM) of ZnOQD. The ZnOQD has excellent crystallinity due to the existence of a crystal lattice spacing of $0.28 \mathrm{~nm}$ [18]. Figure $1 \mathrm{~d}$ shows the selected area electron diffraction (SAED) pattern of ZnOQD. The diffraction rings were referred to as (100), (002), (101), (102), (110), (103) and (112) planes, respectively, indicating that the ZnOQD featured a hexagonal wurtzite structure with high crystallinity [11]. 

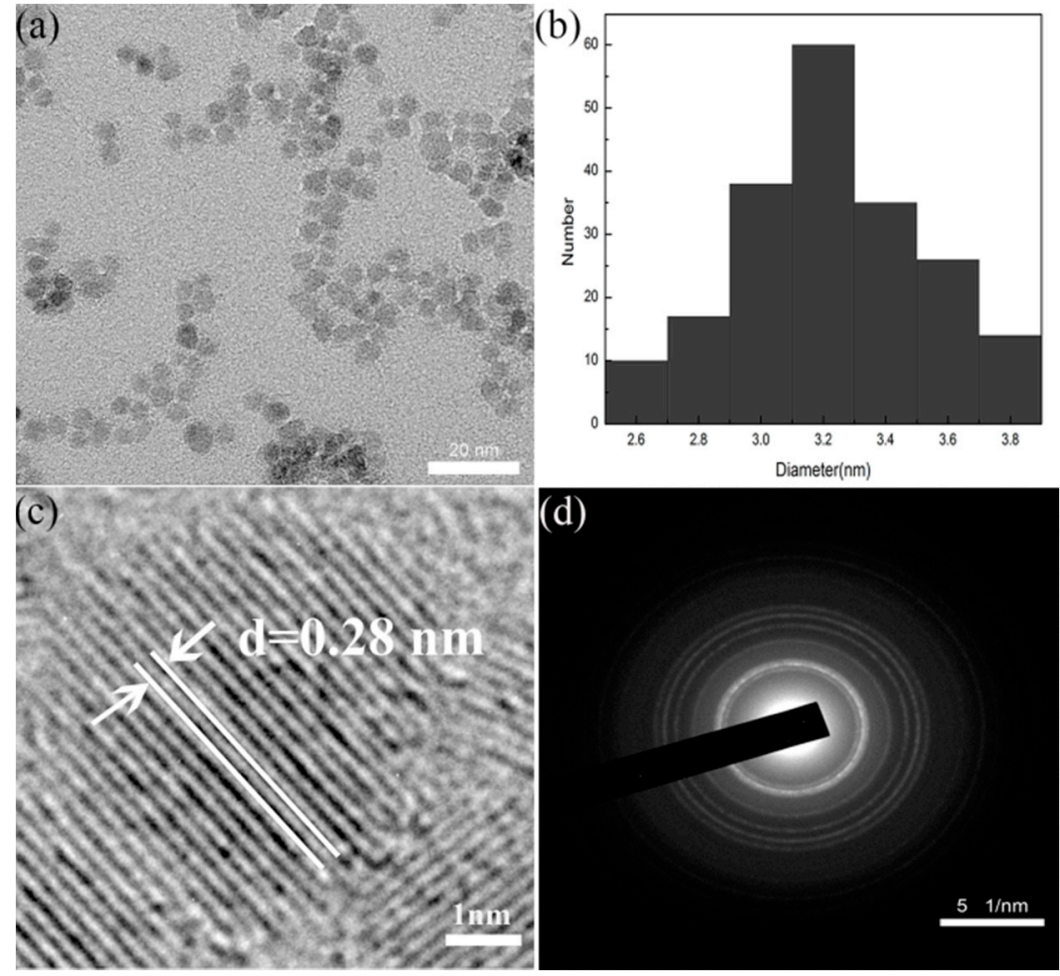

Figure 1. (a) TEM image of ZnOQD, (b) diameter distribution histogram calculated by TEM, (c) enlarged HRTEM (high-resolution transmission electron microscope) image of ZnOQD, (d) SAED (selected area electron diffraction) pattern of ZnOQD.

\section{2. $X R D$ and Photoluminescence Analysis of $\mathrm{ZnOQD}$}

Figure 2a shows the XRD pattern of ZnOQD. From Figure 2a, it can be observed that the dominant peaks of $\mathrm{ZnOQD}$ appear at $2 \theta$ values of $31.9^{\circ}, 36.1^{\circ}, 47.4^{\circ}$ and $56.6^{\circ}$, corresponding to (100), (101), (102), and (110) planes, respectively. Figure $2 \mathrm{~b}$ displays the UV-vis and photoluminescence spectra of $\mathrm{ZnOQD}$, indicating that the $\mathrm{ZnOQD}$ featured good optical properties with a clearly resolved absorption peak at $350 \mathrm{~nm}$ and a symmetrical emission peak at $525 \mathrm{~nm}$. The inset in Figure $2 \mathrm{~b}$ is the digital photo of $\mathrm{ZnOQD}$ aqueous solution, which is excited under $365 \mathrm{~nm}$ UV lamp radiation. Correspondingly, the $\mathrm{ZnOQD}$ aqueous solution exhibits yellow fluorescence due to the quantum-size and surface defects [15].
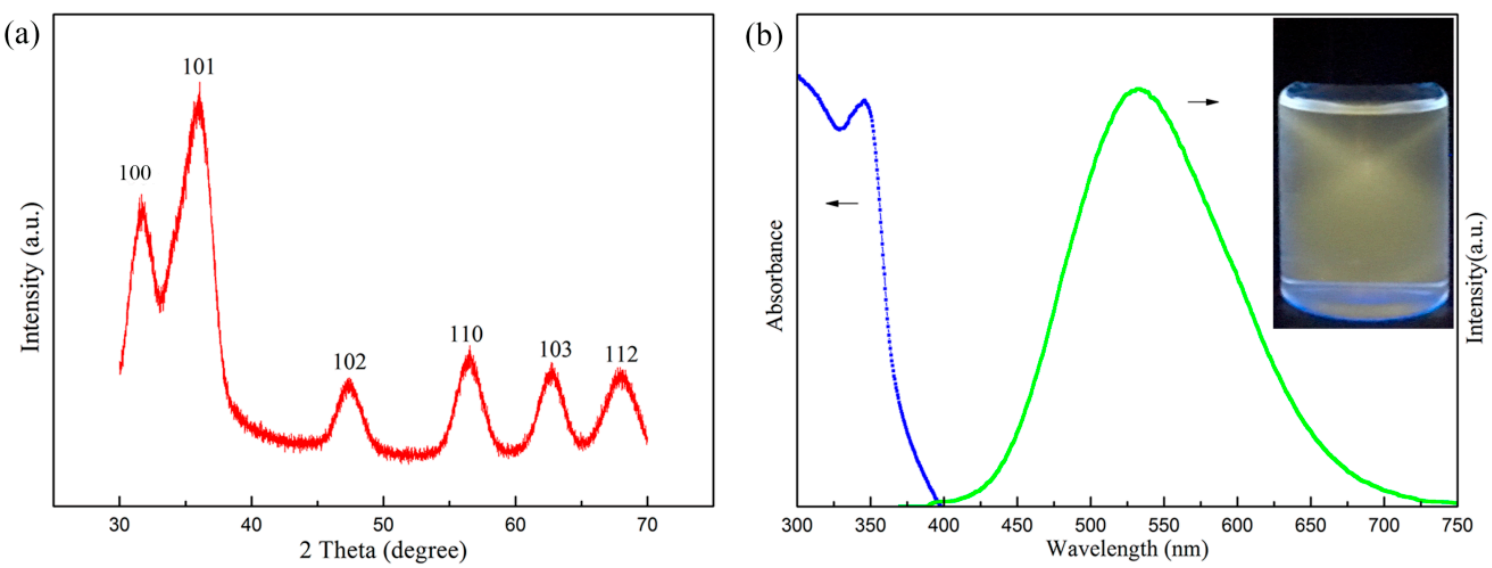

Figure 2. (a) XRD pattern of ZnOQD, (b) UV-vis and photoluminescence spectra of ZnOQD. 


\subsection{Morphology Analysis of $\mathrm{ZnOQD/CMC} \mathrm{Composites}$}

Figure 3a,b give the SEM images of ZnOQD/CMC composites with 5 and 15 wt \% loading of $\mathrm{ZnOQD}$, respectively. From Figure 3a, the CMC-based nanocomposite showed the uniform distribution of $\mathrm{ZnOQD}$. As the ZnOQD content increased from 5 to $15 \mathrm{wt} \%$, a large number of ZnOQD were observed in a $15 \mathrm{wt} \%$ QD-CMC composite, as shown in Figure 3b. Both $5 \mathrm{wt} \%$ QD-CMC and $15 \mathrm{wt} \%$ QD-CMC composites show homogeneous distribution of ZnOQD within the film matrix.
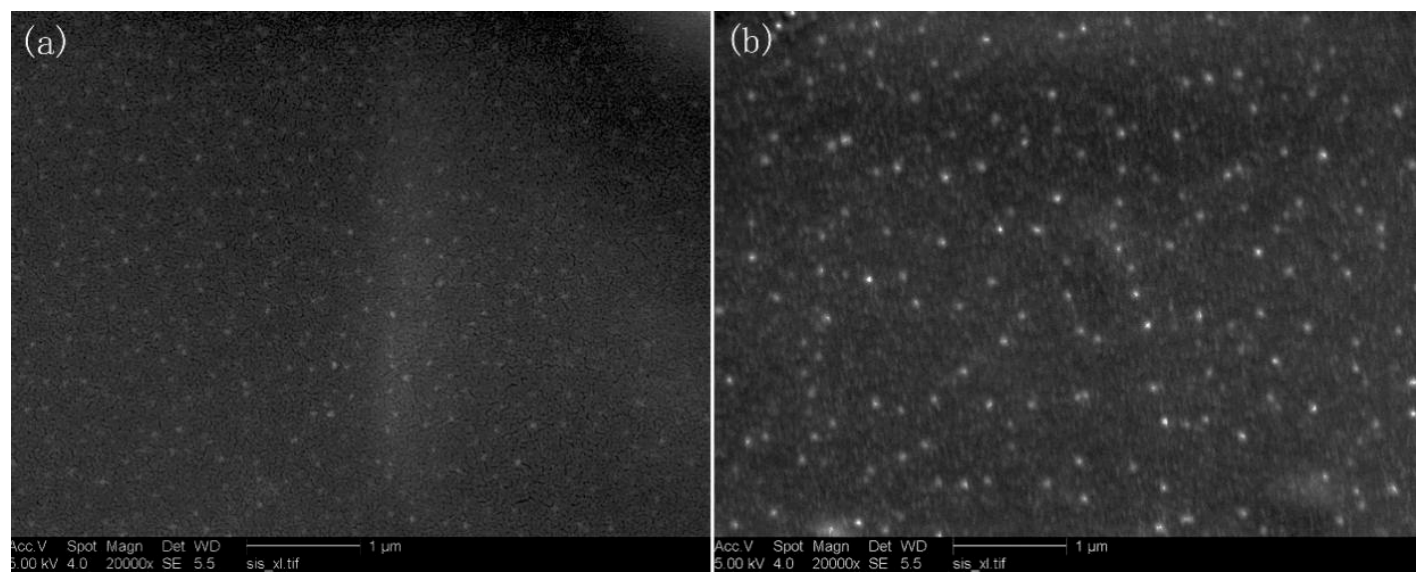

Figure 3. SEM images of (a): $5 \mathrm{wt} \%$ QD-CMC and (b): $15 \mathrm{wt} \%$ QD-CMC composites.

\subsection{XRD Analysis of $\mathrm{ZnOQD/CMC} \mathrm{Composites}$}

Figure 4 shows the XRD pattern of pure CMC and $10 \mathrm{wt} \%$ QD-CMC composites. Both CMC and $10 \mathrm{wt} \%$ QD-CMC composites exhibited the characteristic diffraction peaks of CMC at $2 \theta$ values of $22.2^{\circ}$. Furthermore, a $10 \mathrm{wt} \%$ QD-CMC composite displays (100) and (101) diffraction peaks of ZnOQD at $2 \theta$ values of $31.9^{\circ}$ and $36.1^{\circ}$, respectively. XRD analysis of composites also confirmed that ZnOQD dispersed in the matrix of CMC, which is in accordance with SEM analysis.

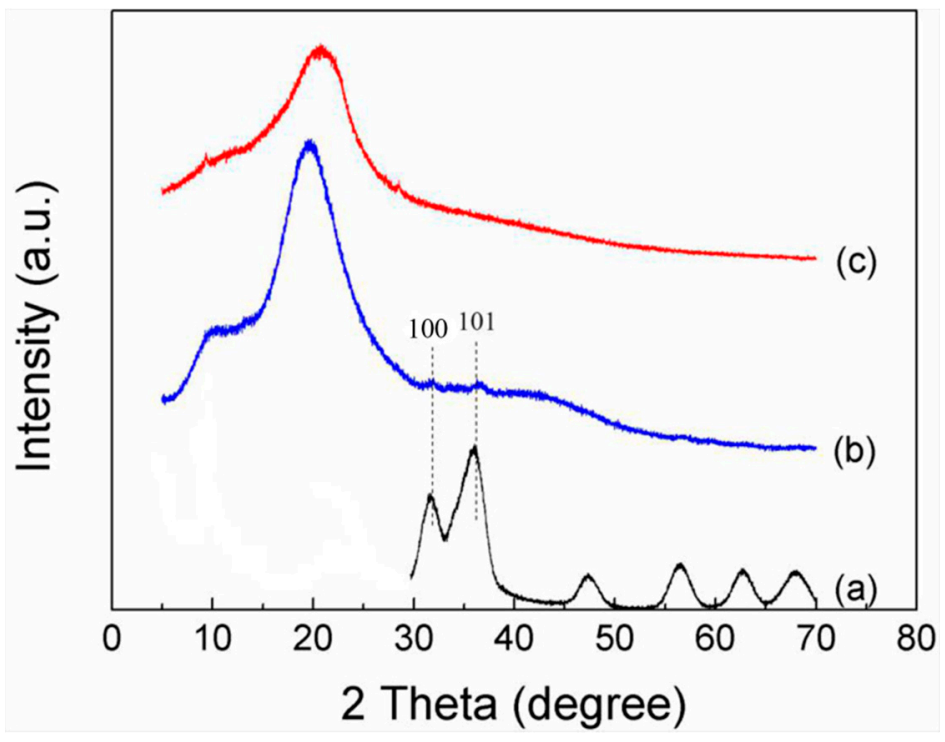

Figure 4. XRD patterns of (a): ZnOQD (b): $10 \mathrm{wt} \%$ QD-CMC composites and (c): pure CMC.

\subsection{Thermal Analysis of CMC and Composites}

Figure $5 a$, b illustrate the TGA and differential thermogravimetry (DTG) curves of CMC and its composites with 5, 10 and $15 \mathrm{wt} \%$ loading of ZnOQD, respectively. As shown in Figure 5a, the first stage with a small weight loss of around $100{ }^{\circ} \mathrm{C}$ corresponds to the evaporation of adsorbed water. 
The second stage with a very fast and significant weight loss, occurring at around $250-340{ }^{\circ} \mathrm{C}$, is owing to the degradation of CMC. The third thermal degradation which started at around $300-380^{\circ} \mathrm{C}$ is due to the pyrolysis of carbonaceous residues at high temperatures. Moreover, the char yield of $5-15 \mathrm{wt} \%$ QD-CMC composites is $42 \%, 40 \%$ and $36 \%$ at $600{ }^{\circ} \mathrm{C}$, respectively. From Figure $5 \mathrm{~b}$, it can be seen that the maximum thermal degradation temperature of $5 \mathrm{wt} \%$ QD-CMC and $10 \mathrm{wt} \%$ QD-CMC composites were both at $278{ }^{\circ} \mathrm{C}$, which was the same as that of pure CMC. The maximum thermal degradation temperature of a $15 \mathrm{wt} \%$ QD-CMC composite was at $269^{\circ} \mathrm{C}$, which was lower than pure CMC film. The reason is that when the ZnOQD loading increases to $15 \mathrm{wt} \%$, a lot of metal and metal oxides promote the catalytic degradation of CMC on heating $[19,20]$. Furthermore, it is shown that the maximum thermal degradation rate gradually decreases with the increase of ZnOQD loading.
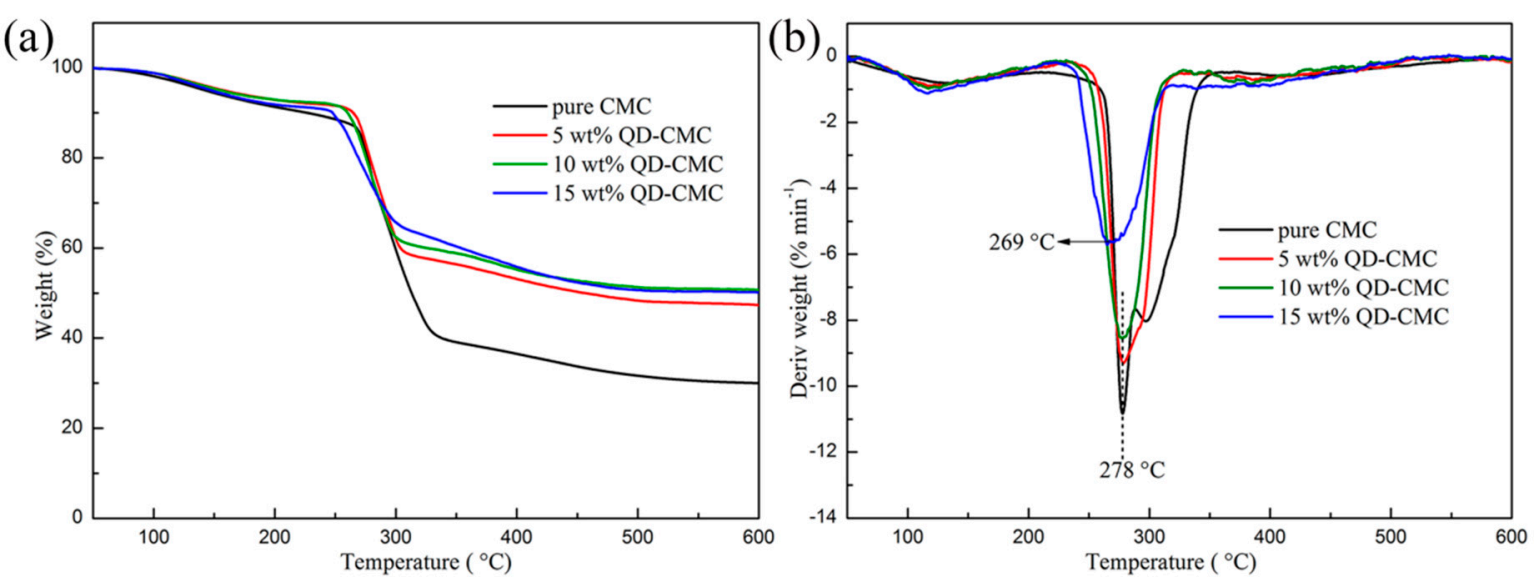

Figure 5. Thermogravimetric analysis (TGA) and differential thermogravimetry (DTG) curves of CMC and composites, (a) TGA curves; and (b) DTG curves.

\subsection{Photoluminescence Analysis of $\mathrm{ZnOQD/CMC} \mathrm{Composites}$}

Figure 6a gives the UV-vis spectra of pure CMC and ZnOQD/CMC composites, respectively. Pure CMC cannot absorb UV radiation. However, the UV absorption band of ZnOQD/CMC almost covers the whole UV range of up to about $375 \mathrm{~nm}$ [21]. Figure $6 \mathrm{~b}$ shows photoluminescence spectra of pure CMC and ZnOQD/CMC composites containing 5, 10, 15 and 20 wt \% ZnOQD excited by $350 \mathrm{~nm}$ wavelength. It clearly exhibits that the pure CMC film features no optical properties. Comparatively, the ZnOQD/CMC composites present significant emission peaks. The maximum emission wavelength appears at $525 \mathrm{~nm}$, which is in accordance with the previously reported ZnOQD [22]. Meanwhile, the results indicate that PL emission intensity is significantly enhanced by adding ZnOQD nanoparticles into a CMC polymer matrix, and a maximum PL emission intensity is reached by adding $15 \mathrm{wt} \% \mathrm{ZnOQD}$. The inset in Figure $6 \mathrm{~b}$ is the digital group of photographs of $5 \mathrm{wt} \%$ QD-CMC, $10 \mathrm{wt} \%$ QD-CMC and $15 \mathrm{wt} \%$ QD-CMC composites in a dark box under $365 \mathrm{~nm}$ UV lamp irradiation. It is obvious that the fluorescent color of composites gradually matches ZnOQD solution with the ZnOQD concentrations increasing to $15 \mathrm{wt} \%$.

Figure $6 \mathrm{c}$ shows the maximum intensity of the photoluminescence spectra of ZnOQD/CMC nanocomposite films with different $\mathrm{ZnOQD}$ concentrations, indicating that the emission intensity does not increase linearly with the increasing loading amount of ZnOQD. It can be found that the emission intensity has an increasing tendency, with the concentration of ZnOQD ranging from 5 to $15 \mathrm{wt} \%$. The obvious increase of emission intensity for a $15 \mathrm{wt} \%$ QD-CMC composite can be interpreted in two ways: Charge trapping effect and chain separation. The ZnOQD can trap electrons and allow more holes to recombine through the interface of CMC and ZnOQD. The exciton formation inside $15 \mathrm{wt} \%$ QD-CMC composite layers is increased in comparison with $5 \mathrm{wt} \%$ QD-CMC and $10 \mathrm{wt} \%$ QD-CMC composites, and thus enhances luminescent properties. However, the emission intensity of the $20 \mathrm{wt} \%$ QD-CMC composite is decreased significantly. A reason for this is that by adding 
too much ZnOQD, most electrons are mainly blocked around the surface of ZnOQD and restrict the recombination population for the ZnOQD/CMC composites [23]. On the other hand, after $15 \mathrm{wt} \%$, $\mathrm{PL}$ emission is partially quenched due to the agglomeration of ZnOQD particles $[1,23,24]$. Therefore, in order to achieve the maximum output efficiency of the ZnOQD/CMC composites in terms of their luminescence levels, the recommended concentration range of ZnOQD is $\sim 15 \mathrm{wt} \%$.
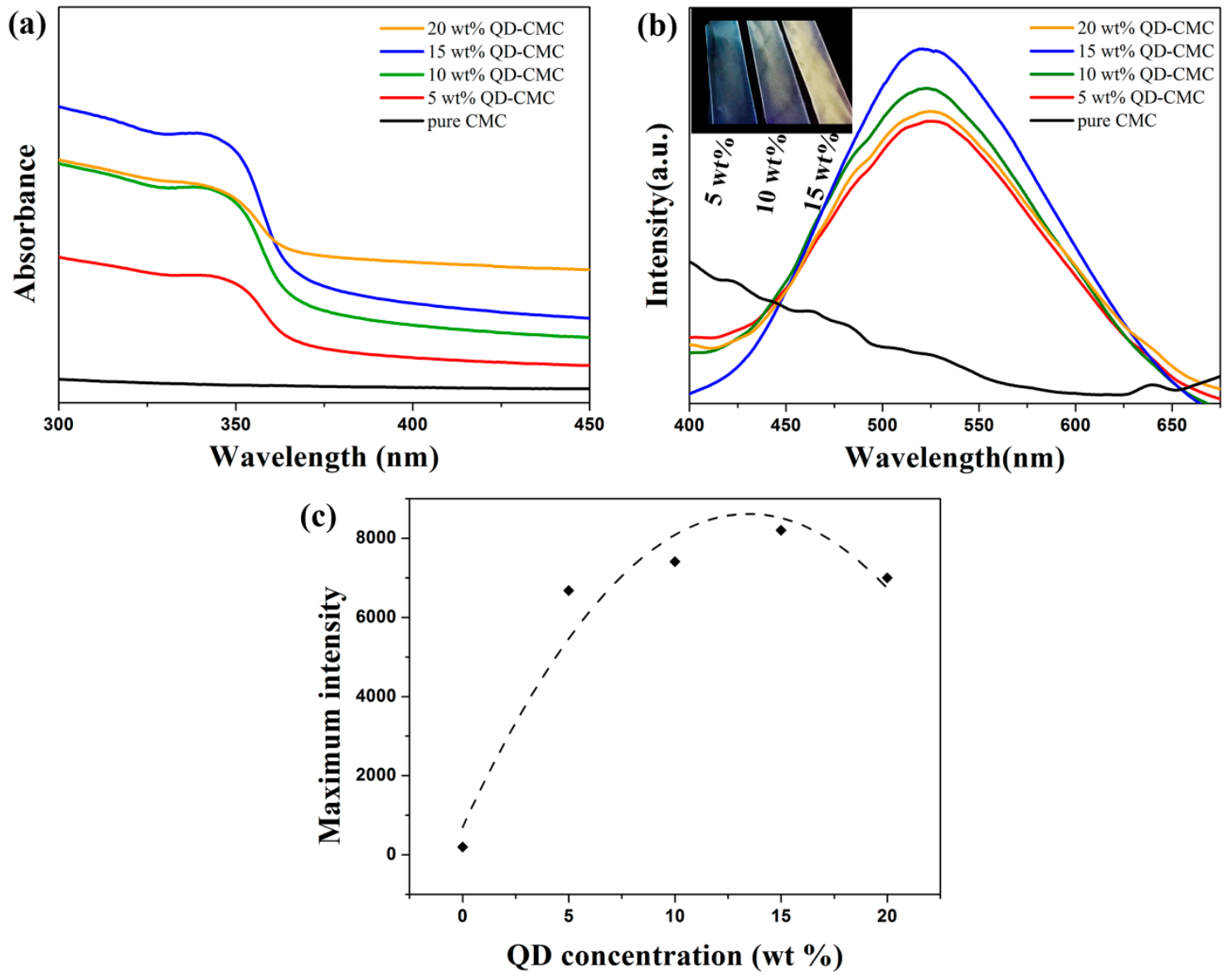

Figure 6. (a) UV-vis spectra and (b) photoluminescence spectra (ex $=350 \mathrm{~nm}$ ) of pure CMC and composites. Inset in (b) is digital photographs of ZnOQD/CMC nanocomposite films under $365 \mathrm{~nm}$ UV lamp. (c) Maximum intensity of the photoluminescence spectra of ZnOQD/CMC nanocomposite films with different $\mathrm{ZnOQD}$ concentrations.

\section{Conclusions}

In summary, ZnOQD was synthesized through a facile ultrasound assisted sol-gel method in a short time. The ZnOQD/CMC composite films were successfully fabricated via an environmentally friendly route. We demonstrated the good optical properties of ZnOQD/CMC composites containing 15 wt \% ZnOQD. In particular, the ZnOQD/CMC composites can absorb the UV radiation of wavelengths less than $375 \mathrm{~nm}$. Therefore, this work raised exciting opportunities of ZnOQD/CMC composites for UV-shielding and optical applications.

Author Contributions: The author W.L. conceived and designed the experiments; W.L. and T.L. analyzed the data and wrote the paper; T.L. and Y.J. performed the experiments; and L.B. contributed data analysis work and revision of this paper.

Acknowledgments: This study was financially supported by the Fundamental Research Funds for the Central Universities (No. 2572017CB24), and the Postdoctoral Scientific Research Starting Foundation of Heilongjiang Province (No. LBH-Q17004).

Conflicts of Interest: The authors declare no conflicts of interest. 


\section{References}

1. Kovalchuk, A.; Huang, K.; Xiang, C.; Martí, A.A.; Tour, J.M. Luminescent Polymer Composite Films Containing Coal-Derived Graphene Quantum Dots. ACS Appl. Mater. Interfaces 2015, 7, 26063-26068. [CrossRef] [PubMed]

2. Chen, T.; Liu, B.; Chen, T.; Liu, B. Dielectric properties of graphene quantum dot-cobalt ferrite-poly(vinylidene fluoride) ternary composites. Mater. Lett. 2017, 209, 163-166. [CrossRef]

3. Koushesh, S.M.; Amini, R. Nano-ZnO/carboxymethyl cellulose-based active coating impact on ready-to-use pomegranate during cold storage. Food Chem. 2017, 232, 721-726. [CrossRef] [PubMed]

4. Youssef, A.M.; Elsayed, S.M.; Elsayed, H.S.; Salama, H.H.; Dufresne, A. Enhancement of Egyptian soft white cheese shelf life using a novel chitosan/carboxymethyl cellulose/zinc oxide bionanocomposite film. Carbohydr. Polym. 2016, 151, 9-19. [CrossRef] [PubMed]

5. Zhang, Q.; Ying, Q.; Huang, X.; Li, C.; Xue, J. Synthesis of Single-walled Carbon Nanotubes-Chitosan Nanocomposites for the Development of an Electrochemical Biosensor for Serum Leptin Detection. Mater. Lett. 2017, 211, 348-351. [CrossRef]

6. Zhu, T.; Yang, C.; Chen, S.; Li, W.; Lou, J.; Wang, J. A facile approach to prepare shell/core nanofibers for drug controlled release. Mater. Lett. 2015, 150, 52-54. [CrossRef]

7. Zhong, Q.; Tian, J.; Liu, T.; Guo, Z.; Ding, S.; Li, H. Preparation and antibacterial properties of carboxymethyl chitosan/ZnO nanocomposite microspheres with enhanced biocompatibility. Mater. Lett. 2017, 212, 58-61. [CrossRef]

8. Roy, N.; Saha, N.; Kitano, T.; Saha, P. Biodegradation of PVP-CMC hydrogel film: A useful food packaging material. Carbohydr. Polym. 2012, 89, 346-353. [CrossRef] [PubMed]

9. Kanmani, P.; Rhim, J.W. Properties and characterization of bionanocomposite films prepared with various biopolymers and ZnO nanoparticles. Carbohydr. Polym. 2014, 106, 190-199. [CrossRef] [PubMed]

10. Noshirvani, N.; Ghanbarzadeh, B.; Mokarram, R.R.; Hashemi, M.; Coma, V. Preparation and characterization of active emulsified films based on chitosan-carboxymethyl cellulose containing zinc oxide nano particles. Int. J. Biol. Macromol. 2017, 99, 530-538. [CrossRef] [PubMed]

11. Fu, Y.S.; Du, X.W.; Kulinich, S.A.; Qiu, J.S.; Qin, W.J.; Li, R.; Sun, J.; Liu, J. Stable Aqueous Dispersion of ZnO Quantum Dots with Strong Blue Emission via Simple Solution Route. J. Am. Chem. Soc. 2007, 129, 16029-16033. [CrossRef] [PubMed]

12. Pan, J.; Zhang, X.; Zhao, C.; Xie, S.; Zheng, Y.; Cui, C.; Li, C. The flexible-transparent photosensitive films of cotton cellulose framework of carbon quantum dots/ZnO. Mater. Lett. 2017, 211, 289-292. [CrossRef]

13. Matsuyama, K.; Mishima, K.; Kato, T.; Irie, K.; Mishima, K. Transparent polymeric hybrid film of ZnO nanoparticle quantum dots and PMMA with high luminescence and tunable emission color. J. Colloid Interface Sci. 2012, 367, 171-177. [CrossRef] [PubMed]

14. Xiong, H.M. ZnO Nanoparticles Applied to Bioimaging and Drug Delivery. Adv. Mater. 2013, 44, 5329-5335. [CrossRef] [PubMed]

15. Tang, X.; Choo, E.S.G.; Li, L.; Ding, J.; Xue, J. Synthesis of ZnO Nanoparticles with Tunable Emission Colors and Their Cell Labeling Applications. Chem. Mater. 2010, 22, 3383-3388. [CrossRef]

16. Nguyen, D.-V.; Jiang, S.; He, C.; Lin, Z.; Lin, N.; Nguyen, A.-T.; Kang, L.; Han, M.-Y.; Liu, X.-Y. Elevating Biomedical Performance of $\mathrm{ZnO} / \mathrm{SiO} 2 @$ Amorphous Calcium Phosphate-Bioinspiration Making Possible the Impossible. Adv. Funct. Mater. 2016, 26, 6921-6929. [CrossRef]

17. Zhang, Y.; Zhuang, S.; Xu, X.; Hu, J. Transparent and UV-shielding ZnO@PMMA nanocomposite films. Opt. Mater. 2013, 36, 169-172. [CrossRef]

18. Joshi, P.; Ansari, Z.A.; Singh, S.P.; Shanker, V. Synthesis and Characterization of Highly Fluorescent Water Dispersible ZnO Quantum Dots. Adv. Sci. Lett. 2009, 2, 360-363. [CrossRef]

19. Wang, L.; Xu, M.; Shi, B.; Li, B. Flame Retardance and Smoke Suppression of CFA/APP/LDHs/EVA Composite. Appl. Sci. 2016, 6, 255. [CrossRef]

20. Wang, L.; Zhang, M.; Zhou, B. Thermal Stability, Combustion Behavior, and Mechanical Property in a Flame-Retardant Polypropylene System. Appl. Sci. 2017, 7, 55. [CrossRef]

21. Sato, M.; Kawata, A.; Morito, S.; Sato, Y.; Yamaguchi, I. Preparation and properties of polymer/zinc oxide nanocomposites using functionalized zinc oxide quantum dots. Eur. Polym. J. 2008, 44, 3430-3438. [CrossRef] 
22. Zhang, Z.; Shao, C.; Gao, F.; Li, X.; Liu, Y. Enhanced ultraviolet emission from highly dispersed ZnO quantum dots embedded in poly(vinyl pyrrolidone) electrospun nanofibers. J. Colloid Interface Sci. 2010, 347, 215-220. [CrossRef] [PubMed]

23. Zou, J.P.; Le Rendu, P.; Musa, I.; Yang, S.H.; Dan, Y.; That, C.T.; Nguyen, T.P. Investigation of the optical properties of polyfluorene/ZnO nanocomposites. Thin Solid Films 2011, 519, 3997-4003. [CrossRef]

24. Balen, R.; da Costa, W.V.; de Lara Andrade, J.; Piai, J.F.; Muniz, E.C.; Companhoni, M.V.; Nakamura, T.U.; Lima, S.M.; da Cunha Andrade, L.H.; Bittencourt, P.R.S.; et al. Structural, thermal, optical properties and cytotoxicity of PMMA/ZnO fibers and films: Potential application in tissue engineering. Appl. Surf. Sci. 2016, 385, 257-267. [CrossRef]

(C) 2018 by the authors. Licensee MDPI, Basel, Switzerland. This article is an open access article distributed under the terms and conditions of the Creative Commons Attribution (CC BY) license (http:/ / creativecommons.org/licenses/by/4.0/). 